\title{
Combined effect of chromaticity and feedback on transverse head-tail instability
}

\author{
Victor Smaluk@, Gabriele Bassi, Alexei Blednykh, and Aamna Khan \\ Brookhaven National Laboratory, Upton, New York 11973, USA
}

(Received 12 December 2020; accepted 19 April 2021; published 18 May 2021)

\begin{abstract}
The resonant interaction of a bunched particle beam with short-range wakefields is the cause of head-tail instability, which is a major limitation for single-bunch beam intensity in circular accelerators. There are several processes that suppress this type of instability, such as fast chromatic damping and Landau damping due to machine nonlinearity. Beam-based feedback systems (transverse dampers) provide active suppression of the beam oscillations. The combined effect of the transverse dampers and chromaticity is discussed in this article. A brief overview of theoretical and experimental studies of the head-tail instability and its mitigation is presented. Results of experimental studies of the transverse mode coupling carried out at National Synchrotron Light Source-II are compared with the theoretical model.
\end{abstract}

DOI: 10.1103/PhysRevAccelBeams.24.054401

\section{INTRODUCTION}

Collective instabilities of particle beams in circular accelerators have been actively studied since the early 1960s. One of the first collective instabilities discovered and studied was likely the coherent instability caused by the beam interaction with the resistive-wall impedance of the vacuum chamber. This instability was observed in the $50-\mathrm{MeV}$ electron accelerator built as a test facility by Midwestern Universities Research Association (MURA, USA) [1], the Princeton-Stanford 500-MeV electronelectron collider (Stanford, USA) [2], and in the Cosmotron 3.3-GeV proton synchrotron (Brookhaven National Laboratory, USA) [3].

The cause of this instability was understood at that time: "the beam became unstable due to a coherent vertical oscillation" and the reason was identified as "interaction of the beam and the walls of the vacuum chamber". For a coasting beam, the theoretical explanation of the instability was proposed and the stabilizing mechanisms were examined by means of the Vlasov equation [4], with the conclusion that increased spread of betatron frequencies among the beam particles can prevent the instability. Interesting to note, the idea of active suppression of the instability by a feedback system was proposed and realized in a very short time after the first observation of the instability [3].

\footnotetext{
*smaluk@bnl.gov
}

Published by the American Physical Society under the terms of the Creative Commons Attribution 4.0 International license. Further distribution of this work must maintain attribution to the author(s) and the published article's title, journal citation, and DOI.
For azimuthally bunched beams, the transverse coupledbunch instability caused by the beam interaction with resistive-wall impedance was theoretically studied using the rigid-bunch model [5]. The modes of oscillation, stability criteria, and the small-amplitude growth rates were derived by solving the eigenvalue problem. For the alternating gradient synchrotron at BNL, the intensitydependent eigenvalues were calculated by a dedicated computer program. Stabilization of the coherent transverse instability by Landau damping caused by the machine nonlinearity was explored in the Institute of Nuclear Physics (Novosibirsk, USSR) for a short single bunch [6].

Since the transverse resistive-wall impedance is narrow band and maximal at low frequencies, it causes the bunchto-bunch interaction (or turn-by-turn interaction of the same bunch in short-circumference machines) via longrange wakefields. The other possible reasons for multibunch instabilities are the beam interaction with narrow band impedance of higher-order modes of accelerating $\mathrm{rf}$ cavities, or with the ions of residual gas. In modern highintensity storage rings, the multibunch instabilities usually affect particle beams with a multibunch filling pattern. Now, almost every synchrotron light source is equipped with a bunch-by-bunch feedback system, which effectively damps this instability.

Unlike the bunch-to-bunch interaction via long-range wakefields, the interaction of a single bunch with shortrange transverse wakefields (broadband impedance) results in head-tail instability. The wakefields induced by the head of a bunch act on the particles of its tail; the head and tail of the bunch interchange periodically due to synchrotron oscillations; the instability occurs if certain conditions of resonance excitation exist. Suppression of the single-bunch instability by feedback was not so straightforward because 
the motion is not fully coherent, this topic is discussed in detail in the next section.

The single-bunch head-tail effect was observed in the VEPP-2 (Russian acronym for "colliding electron-positron beams) electron-positron collider in the Institute of Nuclear Physics (Novosibirsk) [7], in the Orsay Collider Ring ACO (French acronym for "Anneau de Collisions d'Orsay", France), and ADONE (Italian "big AdA", an acronym for "Anello di Accumulazione") electron-positron collider in Frascati (Italy) [8]. The fast damping of coherent betatron oscillations, as well as the transverse head-tail instabilities, was experimentally studied with varied beam current. As it was found, the fast damping could not be explained by resistive walls, it was caused by the beam interaction with the broadband impedance of electrostatic $e^{+} / e^{-}$separators. At the VEPP-2 collider, both horizontal and vertical head-tail instabilities were observed and the horizontal instability was successfully suppressed by an analog feedback system: the input signal was taken from a stripline pickup, amplified, and sent to the stripline kicker. A phase shifter was used in the circuit to tune the kicker signal phase [7].

Early studies of head-tail instability are summarized and discussed in detail in the review [9] presented at the Particle Accelerator Conference in 1969. The results of experimental studies of the feedback performance with varied chromaticity carried out at the ADONE storage ring are compared with analytical estimations. The first theoretical explanations of the head-tail effect published in Refs. $[8,10]$ include two-particle and multiparticle models, as well as the formulation and the solution of an eigenvalue problem to determine the growth rates and frequency shifts of the head-tail modes.

Later, advanced theories were developed using a number of approaches, such as macroparticle models, linearizing the Vlasov equation, applying perturbation theory [11-21]. These comprehensive studies cover almost all aspects of the head-tail effect, including mode coupling, various impedance models, chromaticity, and Landau damping. The multimode analysis of eigenvalue problems is efficient because only the lowest modes are essential.

\section{TRANSVERSE FEEDBACK (DAMPER)}

Transverse feedback systems (dampers) were proposed to cure the beam instabilities almost immediately after they were observed. The idea is straightforward: coherent beam oscillations are measured by a beam position monitor (BPM) (capacitive pickup, stripline), and the signal with proper amplification and phase shift is used to drive a highvoltage rf amplifier connected to a stripline kicker, which deflects the beam to damp the oscillations.

A detailed description of the transverse damper developed and commissioned at SPEAR-II (Stanford Positron Electron Accelerating Ring II, USA) is published in Ref. [22], including design principles, circuit analysis, and results of beam tests. For zero chromaticity, the SPEAR-II damper helped to increase the injected beam current by a factor of 5 . The transverse feedback systems were then installed at many other accelerators, e.g., Super Proton Synchrotron in CERN [23] and the Positron-Electron Tandem Ring Accelerator (PETRA) in Hamburg (Germany) [24], and they were efficient in suppressing coherent instabilities (e.g., coupled-bunch instability caused by resistive walls), as expected. Now, almost all electron rings are equipped with bunch-bybunch feedback systems [25], which are available as commercial products.

However, it was not clear if the damper was able to suppress the chromatic head-tail instability, which is a combination of coherent and incoherent oscillation modes, and how the chromaticity affects damper performance. At the VEPP-2 electron-positron collider, the transverse instability was eliminated by a feedback system [7]; this is likely one of the first published reports on the damper suppressing the head-tail instability. Successful application of the damper to suppress head-tail instability was also experimentally demonstrated at PETRA [26].

The mode-coupling theory of the fast head-tail (transverse mode coupling) instability was expanded by including feedback [15,18-21], which was introduced as an equivalent transverse impedance and the feedback term was added to the matrix equation. A real feedback system with BPMs and stripline kickers has a limited bandwidth. If the bunch is not very long, which is always true for electron rings, the feedback acts on the beam center of mass only, so the feedback can be modeled by a matrix with a single nonzero element related to the coherent mode.

Resistive feedback was already successfully implemented at several machines to suppress multibunch instabilities. However, resistive feedback was originally considered inadequate in the case of the intrabunch instability, because the particle motion in the bunch is complicated and damping all modes by affecting the center of mass looks unfeasible. Reactive feedback systems were proposed for CERN Large Electron-Positron Collider (LEP) $[15,27,28]$ and SLAC Positron-Electron Project (PEP, USA) [29] to suppress the fast head-tail instability. According to the theory developed for LEP in CERN [15], reactive feedback can increase the threshold beam current up to a factor of 4 and resistive feedback is completely ineffective as a cure for this instability. However, experimental results from PEP [30] unexpectedly demonstrated that resistive feedback with high gain provides a larger increase in the threshold beam current than the reactive feedback. The reactive feedback system proposed in Ref. [28] was implemented at the LEP collider resulting in a moderate increase of the threshold current [31].

A transverse feedback system with a variable phase was installed at the VEPP-4M electron-positron collider (Budker Institute of Nuclear Physics, Novosibirsk) and the efficiency of both reactive and resistive feedback was 


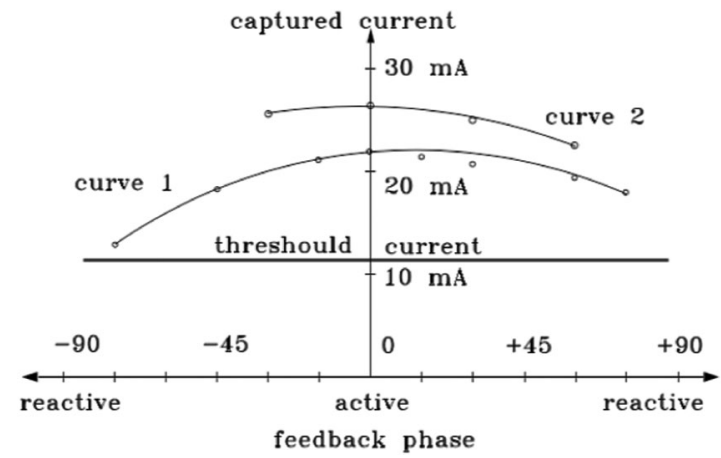

FIG. 1. Single-bunch beam current injected in VEPP-4M as a function of the feedback phase.

experimentally tested [32]. The optimum feedback phase was found closer to $0^{\circ}$ (resistive feedback) than to $90^{\circ}$ (reactive feedback), as shown in Fig. 1. The data corresponding to curves 1 and 2 were measured with different chromaticity values, so this was an experimental confirmation of the combined effect of chromaticity and feedback suppressing the instability.

\section{FEEDBACK AND CHROMATICITY}

Mathematical models of the head-tail instability have been developed on the basis of the multimode analysis of the eigenvalue problem taking into account both chromaticity and feedback [18-21]. As concluded in Ref. [19], resistive feedback in combination with negative chromaticity can effectively damp the instability, increasing the threshold beam current by a factor of 3 to 5 with relaxed tolerances of the feedback parameters. The same conclusion was reached in recent simulation studies of damper efficiency for the Advanced Photon Source (Argonne National Laboratory, USA) and the Large Hadron Collider (CERN) [33].

The efficiency of transverse feedback in combination with varied chromaticity was experimentally studied at several accelerator facilities. The results look quite contradictory. A few experimental confirmations of damper efficiency with negative chromaticity were demonstrated. However, experimental results obtained at several electron storage rings show that feedback is even more efficient at positive chromaticity.

One of the first experiments was carried out at the PETRA electron-positron collider. As it was noticed, for positive chromaticity close to zero, the threshold current increased by about $25 \%$ [26]. With the negative chromaticity of -4.5 and feedback on, the maximum bunch current was more than $6 \mathrm{~mA}$, whereas it was limited to $0.3 \mathrm{~mA}$ without feedback [24].

The feedback performance was studied at the European Synchrotron Radiation Facility (ESRF, France) with positive chromaticity varied up to high values (about 12) [34]. The feedback gain required at low chromaticity to exceed
$15 \mathrm{~mA}$ of bunch current was reduced to almost zero when chromaticity was increased. The threshold single-bunch current was about $1 \mathrm{~mA}$ with zero chromaticity. For regular operations of ESRF, the transverse instability limiting the single-bunch intensity is suppressed by increasing the vertical chromaticity to large positive values.

At the ELETTRA light source (Sincrotrone Trieste, Italy), increasing the chromaticity did not improve the machine's performance by much [35]. A small improvement was observed using the transverse multibunch feedback at positive chromaticity. With negative chromaticity and the feedback, the maximum stable current exceeding the $10 \mathrm{~mA}$ limit by $50 \%$ was achieved but could not be easily reproduced. Usually, the beam current saturates between 6 and $10 \mathrm{~mA}$. Switching the feedback off always causes the beam current to drop below the threshold. Operating with negative chromaticity and transverse feedback in the single- or 4-bunch mode, the beam was very stable at all currents, unlike the operation at positive chromaticity.

A feedback system developed for the VEPP-4M electronpositron collider provides simultaneous suppression of oscillations of colliding electron and positron bunches using the same kickers and pickups [36]. For the standard injection mode with the beam energy of $1845 \mathrm{MeV}$, vertical chromaticity $\xi_{y}=4$, and horizontal chromaticity $\xi_{x}=2$, the threshold beam current is about $5 \mathrm{~mA}$. The feedback provides a reliable increase of the injected current by a factor of 3. Slowly decreasing the chromaticity leads to excitation of the instability and a beam loss. Increasing the positive chromaticity results in a more stable beam: at the vertical chromaticity of 6 , switching the feedback off does not cause a fast beam loss if there is no other perturbation. With the negative vertical chromaticity of -8 and the feedback on, the injected beam current exceeding $10 \mathrm{~mA}$ was achieved. Switching the feedback off results in beam loss down to $0.3-0.4 \mathrm{~mA}$. Thus at negative chromaticity, the relative increase of the beam current in comparison with the feedback off was large; however, the absolute injected beam current was lower than the current achieved at the positive chromaticity. This is also consistent with the numerical simulation based on multiparticle tracking.

The effects of chromaticity and feedback on the transverse beam instabilities were studied at the National Synchrotron Light Source II (NSLS-II) in Brookhaven National Laboratory [37-40]. The threshold beam current of the transverse single-bunch instability was calculated and measured as a function of chromaticity. The stabilizing effect of positive chromaticity was confirmed, the singlebunch threshold current of about $0.8 \mathrm{~mA}$ was measured at zero chromaticity, and the bunch current of $11 \mathrm{~mA}$ was achieved at chromaticity $\xi_{x}=7.0, \quad \xi_{y}=7.8$ with the reduced gain of resistive feedback.

Experimental studies of feedback efficiency with high positive chromaticity were carried out at Synchrotron 


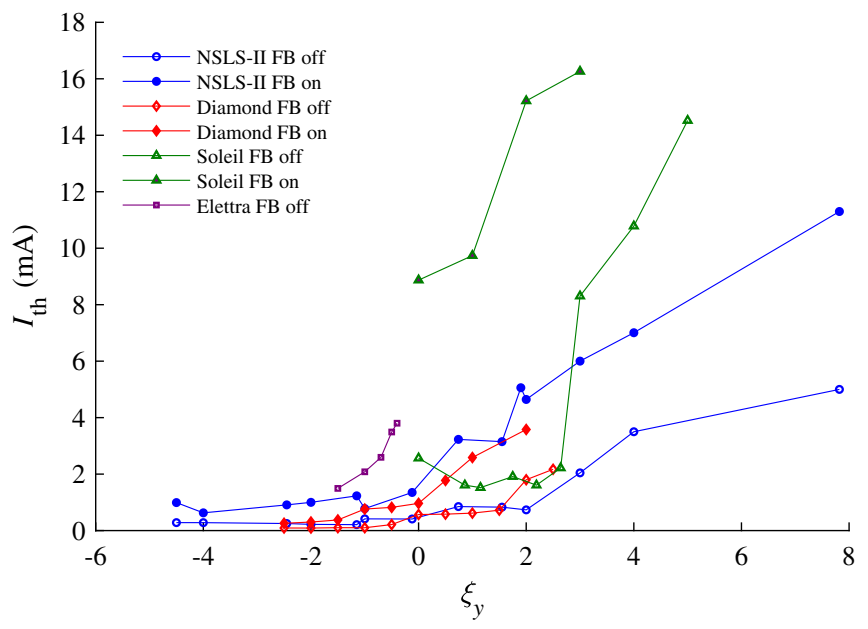

FIG. 2. Measured single-bunch threshold current as a function of chromaticity, with and without feedback.

SOLEIL (France) [41]. Without the feedback, the maximum single-bunch beam current was about $2 \mathrm{~mA}$ with the vertical chromaticity varied from 0 to 3 . At the vertical chromaticity of 3 , a steplike increase of the beam current up to $8 \mathrm{~mA}$ was observed. Further increase of the vertical chromaticity resulted in almost linear growth of the beam current with the chromaticity, reaching $14 \mathrm{~mA}$ at $\xi_{y}=5$. With the resistive feedback on, the beam current was about $8 \mathrm{~mA}$ at $\xi_{y}=0,10 \mathrm{~mA}$ at $\xi_{y}=1$, and $16 \mathrm{~mA}$ at $\xi_{y}=3$.

Single-bunch instabilities were recently studied at Diamond Light Source (UK) with chromaticity varied from -2.5 to 2.5 [42]. Varying the feedback phase from resistive to reactive and intermediate was found helpful in maximizing the achievable beam current. There was no unique phase that works best in all chromaticity regimes. A steplike increase of the beam current with the chromaticity was also observed with and without feedback.

Some of the experimental results discussed above are summarized in Fig. 2. All the data presented in the graph were measured with the positive momentum compaction factor of a few units of $10^{-4}$. Here, it looks like the threshold beam current is higher with positive chromaticity, both with and without feedback.

The other phenomenon that has a significant effect on the instability is the decoherence of the beam oscillation caused by linear and higher-order chromaticity, machine nonlinearity, and beam-beam interaction (in colliders). Any amplitude-dependent or momentum-dependent spread of oscillation frequencies of particles in the bunch results in Landau damping of the oscillations and affects the instability as it was shown at the KEK Photon Factory (Japan) [43], ELETTRA (Italy) [44], and VEPP-4M (Russia) [36].

\section{TRANSVERSE MODE COUPLING}

An effective mathematical model of the head-tail instability with nonzero chromaticity has been developed on the basis of a multimode analysis of the eigenvalue problem [14-21]. For our analysis, we follow the mode-coupling theory $[18,19]$, which takes into account chromaticity and feedback. Starting from the linearized Vlasov equation, the analysis of beam stability is reduced to a system of algebraic equations and the values of the frequency and decrement/increment of the oscillation modes can be found as eigenvalues of the set of equations. According to Refs. $[18,19]$, the symmetric mode expansion is efficient because only a few lowest oscillation modes are significant. This simplification is done with several assumptions: the longitudinal oscillations of the beam particles are much slower than the transverse ones; the longitudinal particle distribution in a bunch is Gaussian; the impedance is broadband; the motion is represented as a sum of oscillation modes $e^{i \Omega t}$, where $\Omega$ is the complex frequency.

In this case, we use a single-mode broadband resonator as the impedance model. The beam-impedance interaction is characterized by the integral parameters, such as the longitudinal loss factor, transverse kick factor, and effective impedance. If the bunch is not much shorter than the vacuum chamber aperture, these parameters can be calculated with acceptable accuracy using the broadband impedance approximated by a single resonator $[45,46]$. We also analyze the horizontal and vertical oscillations separately assuming the motion is transversely uncoupled.

The mode coupling equations we use to describe the data measured at NSLS-II are presented below, the detailed derivation is published in Refs. [18,19]. For a Gaussian beam interacting with a broadband transverse impedance

$$
Z_{\perp \mathrm{BB}}(\omega)=\frac{R_{\perp}}{\frac{\omega}{\omega_{r \perp}}+i Q\left(\frac{\omega^{2}}{\omega_{r \perp}^{2}}-1\right)},
$$

where $\perp$ means $x$ (horizontal) or $y$ (vertical), $\omega_{r \perp}$ is the resonant frequency, and $R_{\perp}$ is the shunt resistance of the broadband $(Q \simeq 1)$ resonator, the set of algebraic equations determining the complex amplitudes $a_{n k}$ of oscillation modes can be written as

$$
\begin{aligned}
& \sum_{n^{\prime}=-\infty}^{\infty} \sum_{k^{\prime}=0}^{\infty}\left(I A_{n k n^{\prime} k^{\prime}}+i n \delta_{n n^{\prime}} \delta_{k k^{\prime}}\right) a_{n^{\prime} k^{\prime}}+i \lambda a_{n k}=0, \\
& n=-\infty, \ldots, \infty ; \quad k=0, \ldots, \infty
\end{aligned}
$$

where $n$ and $k$ are the azimuthal and radial indices, respectively;

$$
\lambda=\frac{\Omega-\omega_{\beta}}{\omega_{s}}
$$

is the normalized complex frequency shift, $\omega_{\beta}$ is the unperturbed betatron frequency, $\omega_{s}$ is the synchrotron frequency, and $\delta_{n n^{\prime}}, \delta_{k k^{\prime}}$ is the Kronecker delta. The dimensionless beam intensity $I$ is normalized in such a 
way that the factor before the sums in Eq. (2) is equal to unity:

$$
I=\frac{I_{b}}{I_{0}}, \quad I_{0}=\frac{4 \pi \frac{E}{e} \omega_{s} \sigma_{s}}{\beta_{\mathrm{av}} R_{\perp} c},
$$

where $I_{b}$ is the beam current, $E$ is the beam energy, $\sigma_{s}$ is the rms bunch length, and $\beta_{\text {av }}$ is the average beta function. The matrix element expressed in terms of the dimensionless integration variable $x=\omega \sigma_{s} / c$ is

$A_{n k n^{\prime} k^{\prime}}=\int_{-\infty}^{\infty} z_{\perp}\left(x \frac{c}{\sigma_{s}}\right) g_{n k}\left(x \frac{c}{\sigma_{s}}+\omega_{\xi}\right) g_{n^{\prime} k^{\prime}}\left(x \frac{c}{\sigma_{s}}+\omega_{\xi}\right) d x$.

Here, $z_{\perp}(\omega)=Z_{\perp \mathrm{BB}}(\omega) / R_{\perp}, \omega_{\xi}=\xi \omega_{0} / \alpha$ is the chromatic frequency, $\xi$ is the chromaticity, $\omega_{0}$ is the revolution frequency, and $\alpha$ is the momentum compaction factor. The functions characterizing oscillation modes of a Gaussian bunch are

$$
g_{n k}(\omega)=\frac{\varepsilon(n)}{(k+|n|) ! k !} e^{-\frac{\omega^{2} \sigma_{s}^{2}}{2 c^{2}}}\left(\frac{\omega \sigma_{s}}{\sqrt{2} c}\right)^{2 k+|n|},
$$

where

$$
\varepsilon(n)=\left\{\begin{array}{ll}
1 & \text { if } n \geq 0 \\
(-1)^{|n|} & \text { if } n<0
\end{array} .\right.
$$

Solving the eigenvalue problem (2) for specific impedance and beam parameters, one can derive the intensitydependent shift $\lambda$ of complex oscillation frequencies for the head-tail modes; $\operatorname{Re} \lambda=\Delta \omega_{\beta} / \omega_{s}$ is the normalized frequency shift per se and $\operatorname{Im} \lambda=2 \pi / \tau \omega_{s}$ is the normalized damping/growth rate. The positive rate corresponds to the damping and the negative rate corresponds to the growth of the mode.

For electron storage rings with short bunches, the intensity-dependent bunch lengthening caused by the combined effect of intrabeam scattering and beam interaction with longitudinal broadband impedance is significant. The bunch length can increase by a factor of 2 per $1 \mathrm{~mA}$ of the single-bunch current or even more. The bunch lengthening weakens collective effects resulting in the head-tail instability, so it is very important to take it into account while solving the eigenmode problem. This is illustrated by Fig. 3 showing ten lowest vertical modes calculated by solving Eq. (2) for NSLS-II. The real and imaginary parts of the normalized complex frequency shift were calculated with the constant zero-current bunch length (left) and with the realistic bunch lengthening (right). As one can see in the graphs on the right side, the beam becomes unstable at a much higher current if the bunch lengthening is taken into account.
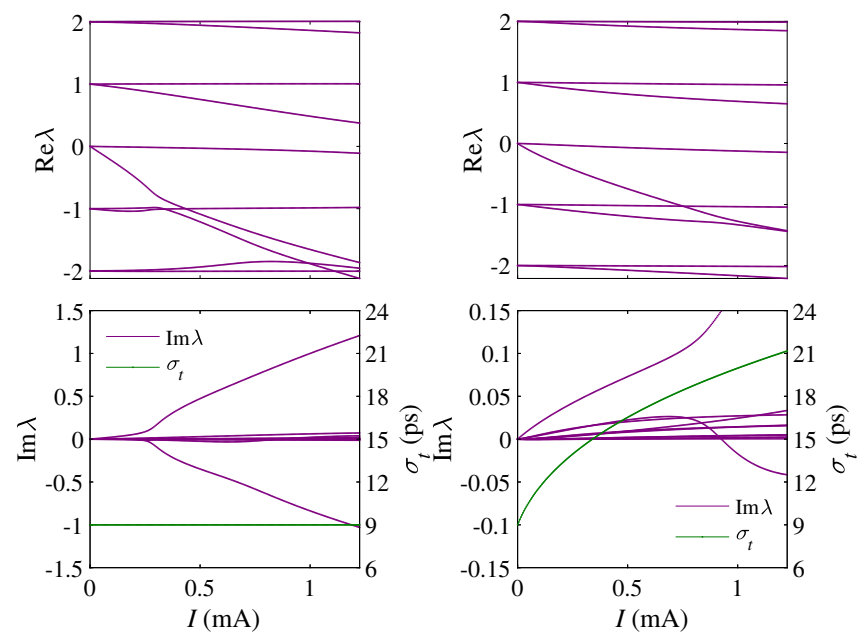

FIG. 3. Effect of bunch lengthening on the frequencies (top) and damping/growth rates (bottom) of the ten lowest vertical modes.

For NSLS-II, the following machine and beam parameters are used: $\nu_{x}=0.217$ and $\nu_{y}=0.262$ are the noninteger parts of the horizontal $(x)$ and vertical $(y)$ betatron tune $\nu_{x, y}=\omega_{x, y} / \omega_{0}, \nu_{s}=\omega_{s} / \omega_{0}=0.008$ is the synchrotron tune, $E=3 \mathrm{GeV}, \beta_{x a v}=13.1 \mathrm{~m}$, and $\beta_{\text {yav }}=15.3 \mathrm{~m}$. Both the horizontal and vertical broadband impedance of the NSLS-II storage ring is approximated by a single broadband resonator (1) with the quality factor $Q=1$, resonance frequency $\omega_{x}=\omega_{y}=2 \pi \cdot 28.6 \mathrm{GHz}$, and shunt resistance $R_{x}=0.3 \mathrm{M} \Omega / \mathrm{m}$ (horizontal), $R_{y}=0.6 \mathrm{M} \Omega / \mathrm{m}$ (vertical). Parameters of the broadband resonators were calculated by the combined fit of the intensity-dependent tune shift and chromatic damping rate measured at a low beam current as described in Ref. [47].

A transverse feedback system can be included in the eigenmode problem as an equivalent impedance $Z_{\mathrm{FB}}(\omega)$. The system of equations with the feedback is reformulated as [19]:

$$
\begin{aligned}
& \sum_{n^{\prime}=-\infty}^{\infty} \sum_{k^{\prime}=0}^{\infty}\left[I\left(A_{n k n^{\prime} k^{\prime}}+f B_{n k n^{\prime} k^{\prime}} \delta_{n 0} \delta_{n^{\prime} 0}\right)+i n \delta_{n n^{\prime}} \delta_{k k^{\prime}}\right] a_{n^{\prime} k^{\prime}} \\
& \quad+i \lambda a_{n k}=0, \\
& n=-\infty, \ldots, \infty ; \quad k=0, \ldots, \infty .
\end{aligned}
$$

Here, the feedback is represented by the matrix $B_{n k n^{\prime} k^{\prime}}$, elements of which are calculated the same way as $A_{n k n^{\prime} k^{\prime}}(5)$ but with the feedback equivalent impedance $Z_{\mathrm{FB}}(\omega)$ replacing the ring impedance $z_{\perp}(\omega)$,

$$
B_{n k n^{\prime} k^{\prime}}=i \frac{\int_{-\infty}^{\infty} Z_{\mathrm{FB}}\left(x \frac{c}{\sigma_{s}}\right) g_{n k}\left(x \frac{c}{\sigma_{s}}+\omega_{\xi}\right) g_{n^{\prime} k^{\prime}}\left(x \frac{c}{\sigma_{s}}+\omega_{\xi}\right) d x}{\int_{-\infty}^{\infty} Z_{\mathrm{FB}}\left(x \frac{c}{\sigma_{s}}\right) g_{00}\left(x \frac{c}{\sigma_{s}}+\omega_{\xi}\right) g_{00}\left(x \frac{c}{\sigma_{s}}+\omega_{\xi}\right) d x} .
$$



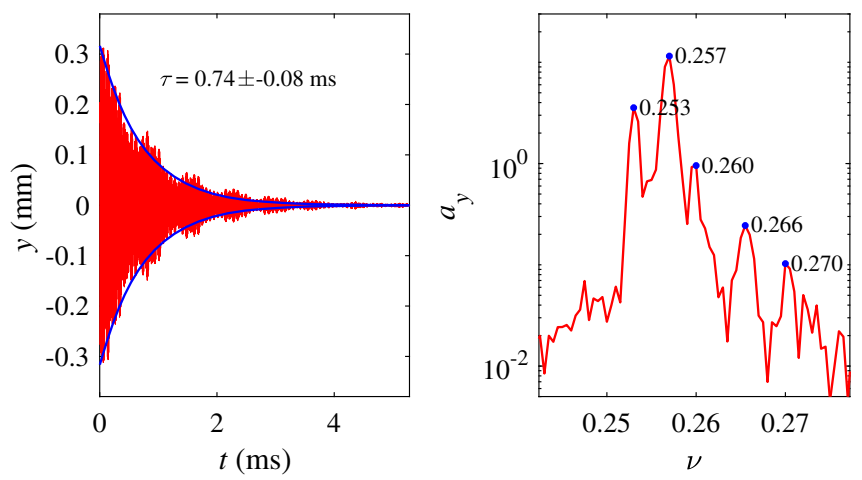

FIG. 4. Vertical turn-by-turn oscillation (left) and its spectrum (right); $\xi_{y}=2.1 ; I_{b}=0.5 \mathrm{~mA}$.

If the real feedback system can interact only with the center-of-mass oscillation of the bunch and does not affect the higher moments, only the terms with $n=0$ and $n^{\prime}=0$ would be nonzero. The feedback parameter $f$ is a complex normalization factor of the feedback matrix elements. The imaginary unit in Eq. (9) means the feedback is resistive when $f$ is real. For resistive feedback, the product If is a decrement introduced by the feedback system into a motion of a rigid bunch with the normalized current $I$ [19].

\section{NSLS-II EXPERIMENTAL RESULTS}

The frequency shifts and damping/growth rates of the low-order horizontal and vertical head-tail modes were measured at NSLS-II with chromaticity varied in the range from -1 to +7 [40]. Coherent beam oscillations were excited by a pulse kicker and measured by button-type BPMs. The measurements were done with and without the bunch-by-bunch feedback system [48]. The oscillation frequencies were obtained by spectral analysis of turnby-turn BPM data using refined discrete Fourier transform [49], the damping/growth rate was obtained by exponential fit of the oscillation envelope. To minimize the oscillation decoherence caused by the betatron tune shift with amplitude, the kick strength was adjusted to excite beam oscillations with the amplitude well below $1 \mathrm{~mm}$. An example of a vertical turn-by-turn oscillation and its spectrum is shown in Fig. 4.

The measured data were compared with the solution of the eigenmode problem (2) using the machine and beam parameters listed above. The set of equations (2) was truncated to five azimuthal modes and two radial ones. Multiparticle numerical simulations were also carried out using the ELEGANT tracking code [50], with and without transverse feedback. The simulations were done using the same machine and beam parameters were used for the mode-coupling equation, the impedance parameters were adjusted to fit the measured data.

Figure 5 shows an example of measured and calculated frequencies (top) and damping/growth rates (bottom) of the

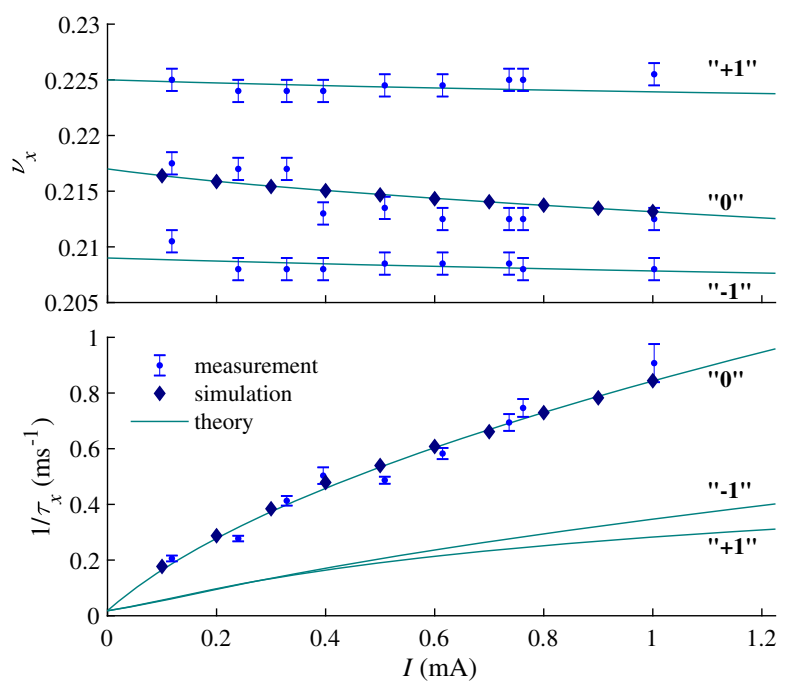

FIG. 5. Frequencies (top) and damping/growth rates (bottom) of horizontal head-tail modes; $\xi_{x}=2.1$; feedback off.

$0,-1$, and +1 horizontal head-tail modes. Figure 6 shows the same parameters of the vertical head-tail modes. The dots with error bars represent the measured data and the solid lines represent the real and imaginary parts of the solution of Eq. (2). The horizontal and vertical linear chromaticities were equal to 2.1 and the bunch-by-bunch feedback was turned off.

With transverse bunch-by-bunch feedback, the set of equations (8) truncated to ten lowest modes was solved with the same machine and beam parameters as for the case without feedback illustrated by Figs. 5 and 6. For these calculations, the equivalent impedance of the NSLS-II bunch-by-bunch feedback system is represented by a simple integrator with the cutoff frequency characterizing the bandwidth of the real system.

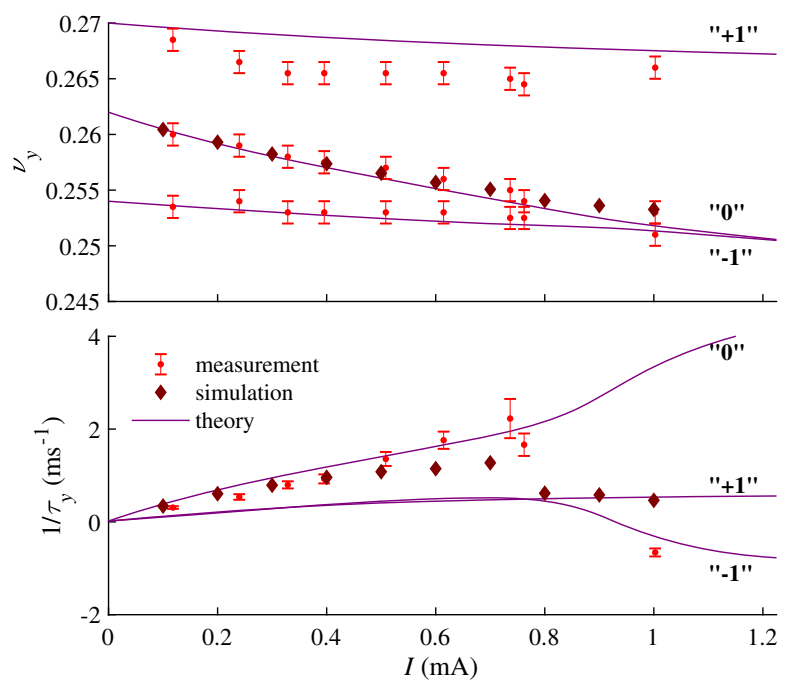

FIG. 6. Frequencies (top) and damping/growth rates (bottom) of vertical head-tail modes; $\xi_{y}=2.1$; feedback off. 

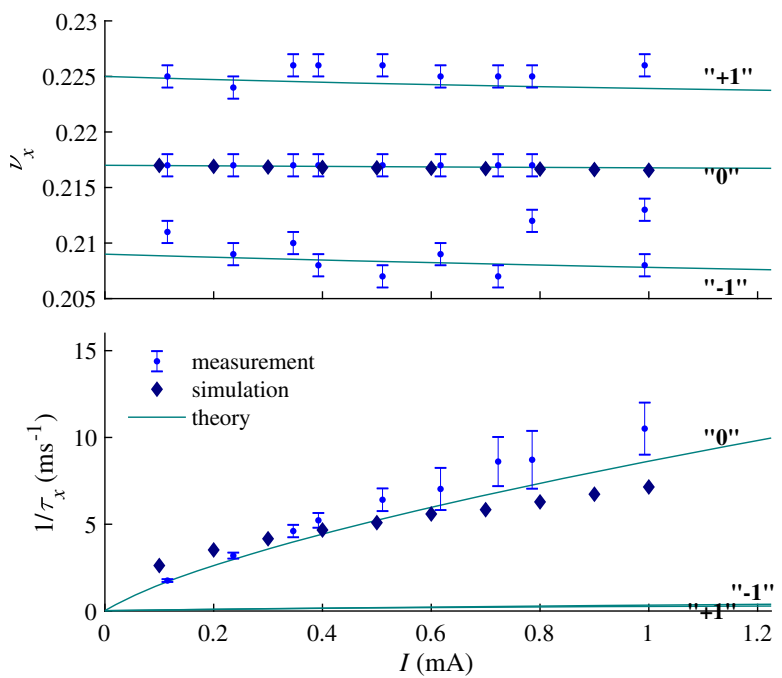

FIG. 7. Frequencies (top) and damping/growth rates (bottom) of horizontal head-tail modes; $\xi_{x}=2.1$; feedback on.

Figure 7 shows an example of measured and calculated frequencies (top) and damping/growth rates (bottom) of the $0,-1$, and +1 horizontal head-tail modes. Figure 8 shows the same parameters of the vertical head-tail modes. As one can see, the feedback decouples the 0 and -1 modes and increases the damping rate by about an order of magnitude. However, the mode-coupling model does not show the saturation-like behavior of the damping rate with the beam current, which is seen in the measurement results and in multiparticle simulations.

For a fixed beam current of $0.2 \mathrm{~mA}$, Fig. 9 shows the measured values of the horizontal (left) and vertical (right) damping rates of the center-of-mass mode vs the chromaticity. There are two series of data, with and without the feedback. The damping rates calculated by solving the

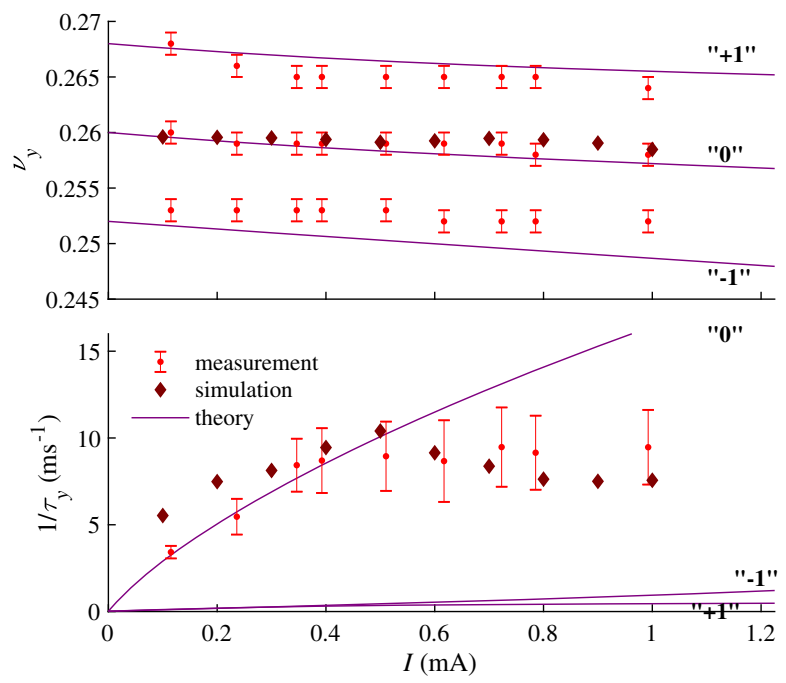

FIG. 8. Frequencies (top) and damping/growth rates (bottom) of vertical head-tail modes; $\xi_{y}=2.1$; feedback on.
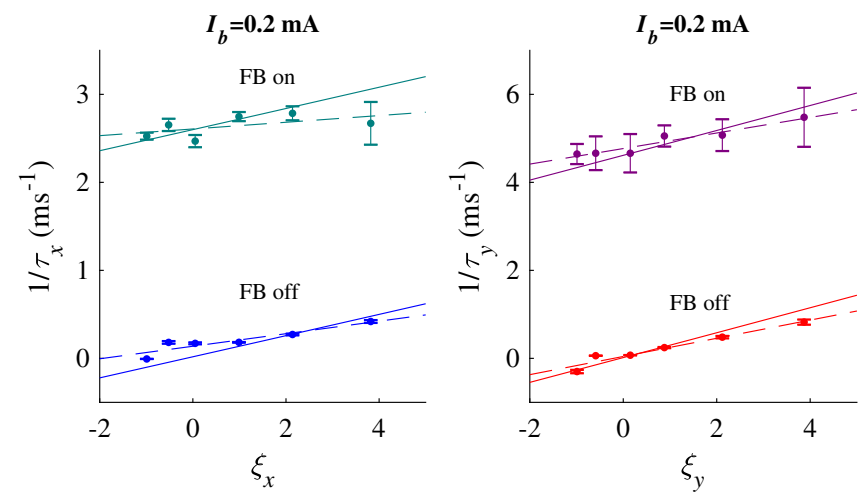

FIG. 9. Horizontal (left) and vertical (right) damping rates of the center-of-mass mode: measured data (dots with error bars), linear fit of the measured data (dashed lines), and the theoretical values (solid lines).

eigenmode problems (2) and (8) are also shown. As one can see, both calculations and experimental data show the same trend with and without the feedback: the damping is stronger with higher chromaticity. Although the relative increase of the instability threshold provided by the feedback at negative chromaticity is large compared to the threshold without feedback, the total injected beam current was higher at the positive chromaticity.

The bunch-by-bunch feedback systems installed at almost all synchrotron light sources are usually designed with the main purpose of suppressing the coupled-bunch instability. So the feedback acts on the center-of-mass motion only. A possible mechanism of the head-tail instability suppression can be explained by the periodic energy exchange between the coherent and incoherent oscillation modes: the feedback can suppress the coherent fraction of oscillation, which always exists due to the chromatic decoherence/recoherence. In the example presented in Fig. 4, one can see multiple modes in the spectrum of the beam oscillation measured by button-type pickups, which are able to detect the beam centroid motion only. Since the growth/damping rates of the head-tail modes strongly decrease with the mode order, it can be more effective to suppress the instability at positive chromaticity, when the damping of the 0 mode is much stronger than the growth of higher modes. On the other hand, the higher modes are stable at negative chromaticity but the growth rate of the 0 mode is large, and much more powerful feedback is required, so the noise sensitivity is higher, which makes the beam unstable. A drawback of high chromaticity is a possible reduction of dynamic aperture and, therefore, of the injection efficiency, so careful optimization of harmonic sextupoles and/or octupoles is needed for stable and reliable operation.

\section{CONCLUSION}

Since the head-tail instability is a severe effect limiting the beam intensity and stability in particle accelerators, the 
instability has been studied theoretically and experimentally ever since it was first observed. The beam-based feedback was proposed as an effective way to suppress the instability; the feedback systems are now implemented at many accelerator facilities. The mode-coupling theory based on multimode analysis of the eigenvalue problem is an effective tool to describe the beam dynamics with the head-tail instability and feedback, taking into account the chromaticity. Starting from the continuum model and the Vlasov equation, the analysis of beam stability is reduced to a system of algebraic equations. Based on the analysis of eigenvalues of the system, it is possible to draw a conclusion about the beam stability. A simplified representation of the machine impedance as a broadband resonator is accurate enough to obtain solutions consistent with experimental data. Taking the intensity-dependent bunch lengthening into account is important for electron machines with short bunches. Both the mode-coupling theory and experiments show that positive chromaticity helps increase the instability threshold even without feedback. Negative chromaticity and feedback result in a significant relative increase in the instability threshold but not in the absolute accumulated beam current. For electron storage rings, operation with negative chromaticity does not look practical, the combination of positive chromaticity and feedback looks more robust. The other factor affecting single-bunch instabilities, which is not taken into account in the mode-coupling model, is the nonlinear spread of oscillation frequencies of particles caused by higher-order chromaticity, machine nonlinearity, and beam-beam interaction in colliders. For accurate analysis of the beam dynamics with nonlinear effects, selfconsistent simulations using advanced computer codes are necessary.

\section{ACKNOWLEDGMENTS}

The authors appreciate the support of NSLS-II engineers and operators in experimental studies. We thank the organizers and participants of the ICFA Mini-Workshop on "Mitigation of Coherent Beam Instabilities in particle accelerators" (Zermatt, 2019) for helpful discussions on the topic. This work was supported by the U.S. Department of Energy under Contract No. DE-SC0012704 and by Brookhaven National Laboratory Directed Research and Development Program, Project No. 20-041.

[1] C. Curtis et al., Beam experiments with the MURA 50 $\mathrm{MeV}$ FFAG accelerator, in Proceedings of HEACC, Dubna, 1963, pp. 124-156, http://inspirehep.net/record/ 918841/files/HEACC63_II_124-156.pdf.

[2] G. K. O'Neill, Storage-ring work at Stanford, in Proceedings of the Summer Study on Storage Rings, Accelerators and Experimentation at Super-High Energies, Upton, 1963, pp. 209-227, https://lss.fnal.gov/conf/C630610/ p209.pdf.
[3] M. Q. Barton, J. G. Cottingham, and A. Tranis, Damping of a resistive wall beam instability in the cosmotron, Rev. Sci. Instrum. 35, 624 (1964).

[4] L. J. Laslett, V. K. Neil, and A. M. Sessler, Transverse resistive instabilities of intense coasting beams in particle accelerators, Rev. Sci. Instrum. 36, 436 (1965).

[5] E. D. Courant and A. M. Sessler, Transverse coherent resistive instabilities of azimuthally bunched beams in particle accelerators, Rev. Sci. Instrum. 37, 1579 (1966).

[6] N. Dikansky and A. Skrinsky, Transverse coherent instability of a bunch of charged particles, At. Energy 21, 176 (1966), http://elib.biblioatom.ru/text/atomnaya-energiya_ t21-3_1966/go,16/.

[7] V. Auslender et al., Study of self-excitation and fast damping of coherent transverse oscillations in the VEPP-2 storage ring, At. Energy 22, 198 (1967), http://elib.biblioatom.ru/ text/atomnaya-energiya_t22-3_1967/go,40/.

[8] C. Pellegrini, On a new instability in electron-positron storage rings (The head-tail effect), Nuovo Cimento A 64 , 447 (1969).

[9] F. Amman, Electron positron storage rings: Status and present limitations, in Proceedings of PAC-1969, Washington, 1969, pp. 1073-1081, https://accelconf.web .cern.ch/accelconf/p69/PDF/PAC1969_1073.PDF.

[10] M. Sands, The head-tail effect: An instability mechanism in storage rings, SLAC Technical Report No. SLAC TN-69008, 1969, https://www.slac.stanford.edu/cgi-bin/getdoc/ slac-tn-69-008.pdf.

[11] F. Sacherer, Methods for computing bunched-beam instabilities, CERN Libraries, Report No. CERN-SI-BR-72-5, 1972, https://cds.cern.ch/record/322545/files/CM-P00063598.pdf.

[12] M. Karliner, Coherent instabilities of a beam in electron storage rings due to interaction with environment, Budker INP Report No. 74-107, 1974, http://www.inp.nsk.su/ images/preprint/1974_107.pdf.

[13] R. Kohaupt, Head-tail turbulence and the transverse PETRA instability, DESY Report No. DESY-80/22, 1980, https://doi.org/10.3204/PUBDB-2017-12952.

[14] Y.H. Chin and K. Satoh, Transverse mode coupling in a bunched beam, in Proceedings of PAC-1983, Santa $\mathrm{Fe}$, 1983, pp. 2566-2568, https://accelconf.web.cern.ch/ accelconf/p83/PDF/PAC1983_2566.PDF.

[15] R. Ruth, Transverse mode coupling with feedback, in Proceedings of HEACC-1983 [Conf. Proc. C 830811, 389 (1984)], http://cds.cern.ch/record/2048901/files/HEACC83_ 389-392.pdf.

[16] N. Dikansky and D. Pestrikov, The Physics of Intense Beams and Storage Rings, Nauka, Moscow, 1989 (AIP Press, New York, 1994).

[17] A. Chao, Physics of Collective Beam Instabilities (Wiley, New York, 1993).

[18] M. Karliner, Theory of a feedback to cure transverse mode coupling instability, Budker INP Report No. 2001-37, 2001, http://www.inp.nsk.su/images/preprint/2001_037.pdf.

[19] M. Karliner and K. Popov, Theory of a feedback to cure transverse mode coupling instability, Nucl. Instrum. Methods Phys. Res., Sect. A 537, 481 (2005).

[20] E. Perevedentsev, Head-tail instability caused by electron cloud, in Proceedings of ECLOUD-02: Mini Workshop on Electron Cloud Simulations for Proton and Positron Beams, 
2002, pp. 171-194, http://cds.cern.ch/record/585578/files/ p171.pdf.

[21] K. Ohmi, F. Zimmermann, and E. Perevedentsev, Wakefield and fast head-tail instability caused by an electron cloud, Phys. Rev. E 65, 016502 (2001).

[22] J.-L. Pellegrin, Transverse oscillations damping with wideband feedback on SPEAR II, IEEE Trans. Nucl. Sci. 22, 1500 (1975).

[23] R. Bossart, I. Burnod, J. Gareyte, B. de Raad, and V. Rossi, The damper for the transverse instabilities of the SPS, IEEE Trans. Nucl. Sci. 26, 3284 (1979).

[24] K. Wille, Damping of coherent transverse oscillations in PETRA, IEEE Trans. Nucl. Sci. 26, 3281 (1979).

[25] T. Nakamura, Transverse and longitudinal bunch-by-bunch feedback for storage rings, in Proceedings of IPAC-2018, Vancouver, 2018, TUZGBD2, http://accelconf.web.cern .ch/AccelConf/ipac2018/papers/tuzgbd2.pdf.

[26] R. Kohaupt, Single beam instabilities in PETRA, IEEE Trans. Nucl. Sci. 26, 3480 (1979).

[27] S. Myers, A first look at the requirements for transverse feedback for the LEP main ring, CERN LEP Report No. 436, 1983, https://cds.cern.ch/record/443365/files/ CM-P00062155.pdf.

[28] V. V. Danilov and E. A. Perevedentsev, Feedback system for elimination of the transverse mode coupling instability, CERN Report No. CERN-SL-93-38-AP, 1993, http://cds .cern.ch/record/253913/files/CM-P00061155.pdf.

[29] S. Myers, Effect of reactive feedback on the transverse mode coupling instability, SLAC Report No. AP-30, 1984.

[30] S. Myers, Stabilization of the fast head-tail instability by feedback, in Proceedings of PAC-1987, Washington, 1987, pp. 503-507, http://accelconf.web.cern.ch/AccelConf/p87/ PDF/PAC1987_0503.PDF.

[31] S. Myers, Conclusions of the fifth LEP performance workshop, CERN Report No. SL-95-10-DI, 1995, https://cds.cern.ch/record/278149/files/sl-95-010.pdf.

[32] M. Karliner, V. Kiselev, A. Medvedko, V. Smaluk, A. Zelenin, and N. Zinevich, The feedback system for elimination the fast head-tail instability at storage ring VEPP-4M, in Proceedings of EPAC-1996, Barcelona, 1996, WEP010G, http://accelconf.web.cern.ch/AccelConf/ e96/PAPERS/WEPG/WEP010G.PDF.

[33] A. Burov, Efficiency of feedbacks for suppression of transverse instabilities of bunched beams, Phys. Rev. Accel. Beams 19, 084402 (2016).

[34] J. Jacob et al., Experimental and theoretical studies of transverse single bunch instabilities at the ESRF, in Proceedings of EPAC, Stockholm, 1998, THP05G, http:// accelconf.web.cern.ch/AccelConf/e98/PAPERS/THP05G .PDF.

[35] E. Karantzoulis and M. Lonza, Transverse head-tail modes elimination with negative chromaticity and the transverse multi-bunch feedback system at ELETTRA, in Proceedings of EPAC, Edinburgh, 2006, THPCH045, http:// accelconf.web.cern.ch/AccelConf/e06/PAPERS/ THPCH045.PDF.

[36] V. Smaluk, D. Sukhanov, V. Oreshonok, V. Cherepanov, and V. Kiselev, Feedback for suppression of single-bunch transverse instability in electron-positron storage rings, J. Instrum. 7, P01007 (2012).
[37] S. Krinsky et al., Collective effects in the NSLS-II storage ring, in Proceedings of PAC-2007, Albuquerque, 2007, TUPMS074, http://accelconf.web.cern.ch/AccelConf/p07/ PAPERS/TUPMS074.PDF.

[38] A. Blednykh et al., First collective effects measurements in NSLS-II with ID's, in Proceedings of IPAC-2015, Richmond, 2015, TUAB2, http://accelconf.web.cern.ch/ AccelConf/IPAC2015/papers/tuab2.pdf.

[39] G. Bassi, A. Blednykh, V. Smaluk, and Z. Yang, A model to simulate the effect of a transverse feedback system on single bunch instability thresholds, in Proceedings of NAPAC-2016, Chicago, 2016, TUPOB45, https://accelconf .web.cern.ch/napac2016/papers/tupob45.pdf.

[40] V. Smaluk, Interplay of transverse damper and head-tail instability, Proceedings of the ICFA Mini-Workshop in Mitigation of Coherent Beam Instabilities in Particle Accelerators, Zermatt, 2019, https://indico.cern.ch/event/ 775147/contributions/3439400/attachments/1915564/ 3166805/Smaluk_-_Interplay_of_transverse_damper_ and_HeadTail_instability.pdf.

[41] R. Nagaoka, Collective effects at SOLEIL, TWIICE-II, Abingdon, 2016, https://indico.cern.ch/event/459623/ contributions/1131140/attachments/1226267/1795307/ Nagaoka_FacilityTalk_SOLEIL.pdf.

[42] E. Koukovini-Platia, A. F. D. Morgan, G. Rehm, and R. Bartolini, Study of single bunch instabilities with transverse feedback at diamond, in Proceedings of IPAC-2017, Copenhagen, 2017, THPVA029, http://accelconf.web.cern .ch/AccelConf/ipac2017/papers/thpva029.pdf.

[43] K. Ohmi and Y. Kobayashi, Head-tail effect due to lattice nonlinearities in storage rings, Phys. Rev. E 59, 1167 (1999).

[44] L. Tosi, V. Smaluk, and E. Karantzoulis, Landau damping via the harmonic sextupole, Phys. Rev. ST Accel. Beams 6, 054401 (2003).

[45] V. Smaluk, Comparison of impedance beam-based measurements and simulations, Beam Tests and Commissioning of Low Emittance Storage Rings, Karlsruhe, 2019, https://indico.cern.ch/event/772326/contributions/ 3283298/attachments/1798073/2932186/Smaluk__Impedance_measurement_computation.pdf.

[46] V. Smaluk, A. Blednykh, G. Bassi, and B. Bacha, Beam-based measurement of broadband longitudinal impedance at NSLS-II, in Proceedings of IPAC-2019, Melbourne, 2019, MOPGW122, https://accelconf.web.cern .ch/ipac2019/papers/mopgw122.pdf.

[47] V. Smaluk, I. Martin, R. Fielder, and R. Bartolini, Beambased model of broad-band impedance of the Diamond Light Source, Phys. Rev. ST Accel. Beams 18, 064401 (2015).

[48] W. Cheng, B. Bacha, D. Teytelman, Y. Hu, H. Xu, and O. Singh, Commissioning of bunch-by-bunch feedback system for NSLS2 storage ring, in Proceedings of IBIC-2014, Monterey, 2014, WEPD27, https://accelconf.web.cern.ch/ IBIC2014/papers/wepd27.pdf.

[49] V. Smaluk, Discrete spectral analysis of beam oscillation, Nucl. Instrum. Methods Phys. Res., Sect. A 578, 306 (2007).

[50] M. Borland, ELEGANT: A flexible SDDS-compliant code for accelerator simulation, Advanced Photon Source Report No. LS-287, 2000, https://publications.anl.gov/ anlpubs/2000/08/36940.pdf. 